\title{
Synergistic antitumor interactions between gemcitabine and clofarabine in human pancreatic cancer cell lines
}

\author{
YINGJIE GUO ${ }^{1}$, XUELIAN XU ${ }^{1}$, WENXIU QI ${ }^{1}$, CHENGZHI XIE $^{1}$, \\ GUAN WANG $^{1}$, AIJUN ZHANG ${ }^{2}$ and YUBIN GE GH,4 $^{1,3,4}$
}

\author{
${ }^{1}$ The State Engineering Laboratory of AIDS Vaccine, College of Life Science, Jilin University, Changchun; \\ ${ }^{2}$ Key Laboratory for Molecular Enzymology and Engineering of the Ministry of Education, Jilin University, \\ Changchun, P.R. China; ${ }^{3}$ Department of Oncology, Wayne State University School of Medicine, \\ Detroit, MI 48201; ${ }^{4}$ Developmental Therapeutics Program, Barbara Ann Karmanos Cancer Institute, \\ Wayne State University School of Medicine, Detroit, MI 48201, USA
}

Received August 30, 2011; Accepted November 30, 2011

DOI: $10.3892 / \mathrm{mmr} .2011 .738$

\begin{abstract}
Pancreatic cancer is a highly malignant disease, with a 5 -year survival rate of less than $4 \%$. Thus, new therapies for this deadly disease are urgently required. In this study, we sought to investigate whether combination treatments with gemcitabine and clofarabine result in synergistic cytotoxic effects against human pancreatic cancer cells. The type and extent of cytotoxic interactions between gemcitabine and clofarabine in three human pancreatic cancer cell lines were determined by MTT assays and standard isobologram analysis. The effects of the two agents on cell cycle distribution and apoptosis were determined by flow cytometry. Clofarabine significantly and synergistically enhanced gemcitabine cytotoxicities in all of the cell lines tested. This was accompanied by a nearly complete $\mathrm{S}$ phase arrest and synergistic induction of apoptosis (cooperativity index $=0.67$ ). Combined treatment of gemcitabine and clofarabine resulted in synergistic cytotoxicities in vitro. Our results strongly suggest potential clinical benefits for using this drug combination to treat pancreatic cancer patients.
\end{abstract}

\section{Introduction}

Pancreatic cancer is a highly malignant disease with a 5-year survival rate of less than $4 \%$ (1). Gemcitabine (2',2'-difluorodeoxycytidine; $\mathrm{dFdC}$ ) is the standard first-line drug used to treat patients with advanced pancreatic cancer (2). However, with a median survival rate of 5.7 months and a 1-year survival rate of $18 \%$, its efficacy remains low $(3,4)$. Therefore, there has been

Correspondence to: Dr Yubin Ge, Department of Oncology, Wayne State University School of Medicine, 110 East Warren Ave., Detroit, MI 48201, USA

E-mail: gey@karmanos.org

Key words: pancreatic cancer, gemcitabine, clofarabine, synergistic antitumor interaction considerable interest in combining gemcitabine with a variety of chemotherapeutic agents to improve its efficacy.

As with other nucleoside analogs, gemcitabine exerts its cytotoxic effects after phosphorylation by deoxycitidine kinase (dCK) to its triphosphate active metabolite, which inhibits both DNA polymerase and ribonucleotide reductase, leading to impaired DNA synthesis and repair, and induces apoptosis (5). Previous studies have shown that the combined effects of many anticancer drugs with gemcitabine are based on their ability to affect DNA damage, cell cycle and apoptosis, or to modulate the metabolism of gemcitabine (6). Positive interactions between gemcitabine and other nucleoside analogs have been reported in several previous studies (7-9). One study showed that gemcitabine and a pyrimidine nucleoside analog, 1- $\beta$-D-arabinofuranosyl cytosine (ara-C), had synergistic antileukemic interactions in vitro and in vivo (8). Another two studies demonstrated that the combination of gemcitabine and a purine nucleoside analog, fludarabine or cladribine, resulted in synergistic cytotoxicities against mesothelioma cell lines or isolated chronic lymphocytic leukemia cells $(7,9)$. These studies suggest that combining two nucleoside analogs may enhance their antitumor activities.

Clofarabine (2-chloro-2'-arabino-fluoro-2'-deoxyadenosine; CAFdA) is a second generation deoxyadenosine analog, developed with the intent of combining the favorable mechanistic properties of cladribine and fludarabine (10). Indeed, the introduction of a fluorine atom at the 2'-arabino position of cladribine decreases susceptibility to enzymatic phosphorolysis by Escherichia coli purine nucleoside phosphorylase and increases the acid stability of clofarabine (10). Therefore, clofarabine has higher bioavailability and anticancer activity than cladribine and fludarabine. Clofarabine has been approved for use in the treatment of relapsed and refractory pediatric acute lymphoblastic leukemia alone or in combination with ara-C (11-13). Moreover, pre-clinical studies have also demonstrated that it has broad and potent activity against solid tumors, including pancreatic cancer (14).

In this study, we hypothesized that clofarabine, analogous to cladribine and fludarabine, potentiates the antitumor 
activity of gemcitabine against pancreatic cancer cells. We demonstrate highly synergistic antitumor activities of combined gemcitabine and clofarabine in three pancreatic cell lines, accompanied by synergistic induction of apoptosis and a nearly complete $\mathrm{S}$ phase arrest. Our results suggest potential clinical benefits for using this drug combination to treat patients with pancreatic cancer.

\section{Materials and methods}

Materials. Gemcitabine hydrochloride $\left(\right.$ Gemzar $^{\circledR}$, Eli Lilly, France; $200 \mathrm{mg} / \mathrm{vial}$ ) was purchased from the Pharmacy of the First Hospital of Jilin University (Changchun, China). Clofarabine was purchased from the Jiangsu Wuyi Pharmaceutical Research \& Development Co. (Jinhua, China). Dulbecco's modified Eagle's medium (DMEM) was purchased from Invitrogen (Carlsbad, CA, USA). Fetal bovine serum (FBS) was purchased from the Hangzhou Sijiqing Biological Engineering Materials Co. (Hangzhou, China). MTT (3-[4, 5-dimethylthiazol-2-yl]-2,5-diphenyl tetrazolium bromide), was purchased from Sigma-Aldrich Inc. (St. Louis, MO, USA). Annexin V-FITC/propidium iodide (PI) kit for measuring apoptosis by flow cytometry was purchased from the Shanghai Yanbin Industrial Science and Technology Co. (Shanghai, China).

Cell culture. The BxPC-3, PANC-1 and CFPAC-1 human pancreatic cancer cell lines were purchased from the Shanghai Cell Bank of Chinese Academy of Sciences (Shanghai, China). The cell lines were cultured in DMEM with $10 \%$ FBS, plus $100 \mathrm{U} / \mathrm{ml}$ penicillin and $100 \mu \mathrm{g} / \mathrm{ml}$ streptomycin, in a $37^{\circ} \mathrm{C}$ humidified atmosphere containing $5 \% \mathrm{CO}_{2} / 95 \%$ air.

In vitro cytotoxicity assays. In vitro gemcitabine and clofarabine cytotoxicities against the pancreatic cancer cell lines were measured using MTT assays, as previously described $(15,16)$. $\mathrm{IC}_{50}$ values were calculated as the drug concentrations necessary to inhibit $50 \%$ proliferation as compared to untreated control cells. The extent and direction of gemcitabine and clofarabine cytotoxic interactions were evaluated as previously described (16-18). Briefly, synergism, additivity or antagonism were quantified by determining the combination index (CI), where $\mathrm{CI}<1, \mathrm{CI}=1$ and $\mathrm{CI}>1$ indicate synergistic, additive and antagonistic effects, respectively. The data are presented as the means \pm standard errors from at least three independent experiments.

Cell cycle analysis. BxPC-3 cells treated with $5 \mathrm{nM}$ gemcitabine or $2.5 \mathrm{nM}$ clofarabine alone or in combination for $120 \mathrm{~h}$ were then harvested and fixed with ice-cold $70 \%$ (v/v) ethanol for $24 \mathrm{~h}$. Following centrifugation at $200 \mathrm{x}$ g for $5 \mathrm{~min}$, the cell pellets were washed with PBS (pH 7.4) and resuspended in PBS containing propidium iodide (PI) $(50 \mu \mathrm{g} / \mathrm{ml})$, Triton X-100 $(0.1 \%$, $\mathrm{v} / \mathrm{v})$ and DNase-free RNase $(1 \mu \mathrm{g} / \mathrm{ml})$. DNA contents were determined by flow cytometry (FACS Calibur; Becton Dickinson, San Jose, CA, USA). Cell cycle analysis carried out with ModFit LT $^{\text {тм}}$ 3.0 DNA analysis software (Becton Dickinson).

Detection of apoptosis by flow cytometry. BxPC-3 cells treated with $5 \mathrm{nM}$ gemcitabine or $2.5 \mathrm{nM}$ clofarabine alone
A

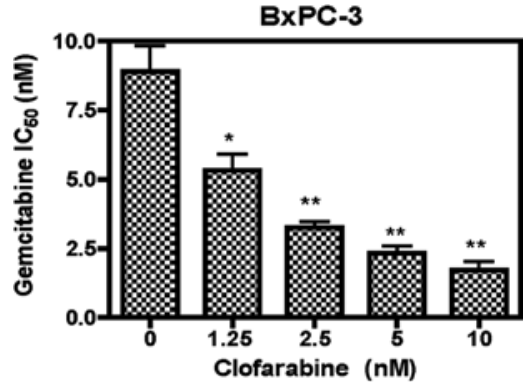

B

PANC-1

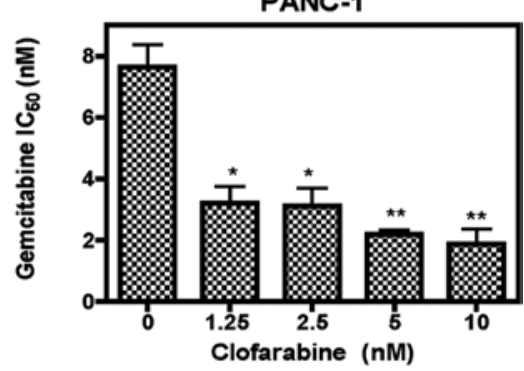

C

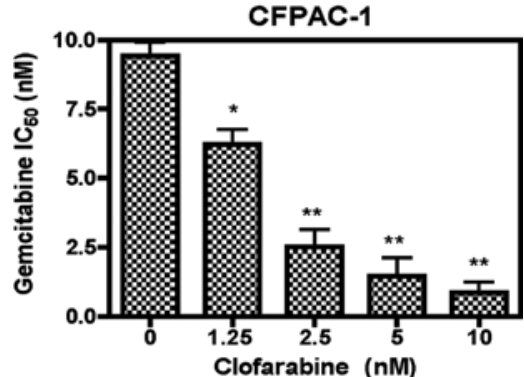

Figure 1. Clofarabine significantly enhanced gemcitabine sensitivities in pancreatic cancer cell lines. Gemcitabine $\mathrm{IC}_{50}$ value for (A) BxPC-3, (B) PANC-1 and (C) CFPAC-1 cells was determined in the presence or absence of clofarabine, treated simultaneously by MTT assays, as described in Materials and methods. ${ }^{*} \mathrm{P}<0.05,{ }^{* *} \mathrm{P}<0.005$.

or their combination for $120 \mathrm{~h}$ were harvested and vigorously pipetted, and samples were taken to determine baseline and drug-induced apoptosis using the Apoptosis Annexin V-FITC/ PI kit, according to the manufacturer's instructions. Apoptotic events were recorded as a combination of Annexin $\mathrm{V}^{+} / \mathrm{PI}^{-}$(early apoptotic) and Annexin $\mathrm{V}^{+} / \mathrm{PI}^{+}$(late apoptotic/dead) events, and the results were expressed as the percentage of Annexin $\mathrm{V}^{+}$cells after subtracting results for untreated cells. Synergy was quantified using the cooperativity index (cooperativity index $=$ sum of apoptosis of the single-agent treatment/apoptosis of the combined treatment). A cooperativity index $<1,=1$ or $>1$ was indicative of synergism, additivity or antagonism, respectively (19).

Statistical analysis. Differences in gemcitabine $\mathrm{IC}_{50}$ values between clofarabine-treated and untreated pancreatic cancer cells were compared using the paired t-test. Statistical analyses were performed with GraphPad Prism 4.0.

\section{Results}

Synergistic cytotoxicities of the combined treatment with gemcitabine and clofarabine in pancreatic cancer cell lines. To explore the possibility of synergistic cytotoxicity of 
Table I. Effects of clofarabine on gemcitabine sensitivities in human pancreatic cancer cell lines.

\begin{tabular}{lccccccr}
\hline Cell line & $\begin{array}{c}\text { Clofarabine } \\
\mathrm{IC}_{50}(\mathrm{nM})\end{array}$ & \multicolumn{5}{c}{ Gemcitabine $\mathrm{IC}_{50}(\mathrm{nM})$} & P-value \\
\cline { 3 - 7 } & & $\begin{array}{c}\text { Clofarabine } \\
0 \mathrm{nM}\end{array}$ & $\begin{array}{c}\text { Clofarabine } \\
1.25 \mathrm{nM}\end{array}$ & $\begin{array}{c}\text { Clofarabine } \\
2.5 \mathrm{nM}\end{array}$ & $\begin{array}{c}\text { Clofarabine } \\
5 \mathrm{nM}\end{array}$ & $\begin{array}{c}\text { Clofarabine } \\
10 \mathrm{nM}\end{array}$ \\
\hline BxPC-3 & $40.7 \pm 2.4$ & $8.9 \pm 0.9$ & $5.3 \pm 0.6(0.63)$ & $3.3 \pm 0.2(0.43)$ & $2.3 \pm 0.3(0.38)$ & $1.7 \pm 0.3(0.44)$ & $<0.05$ \\
PANC-1 & $42.4 \pm 0.7$ & $7.6 \pm 0.7$ & $3.2 \pm 0.5(0.45)$ & $3.1 \pm 0.6(0.47)$ & $2.2 \pm 0.2(0.41)$ & $0.9 \pm 0.5(0.36)$ & $<0.05$ \\
CFPAC-1 & $44.3 \pm 0.9$ & $9.4 \pm 0.5$ & $6.2 \pm 0.5(0.69)$ & $2.5 \pm 0.6(0.33)$ & $1.5 \pm 0.7(0.27)$ & $0.9 \pm 0.4(0.33)$ & $<0.05$ \\
\hline
\end{tabular}

Gemcitabine $\mathrm{IC}_{50}$ values for three cell lines are presented as the means \pm standard error from at least three independent experiments. The values in parentheses represent the CI indices.

gemcitabine in combination with clofarabine, the three human pancreatic cancer cell lines (BxPC-3, PANC-1 and CFPAC-1) were treated with various concentrations of gemcitabine and clofarabine, either alone or in combination, for $120 \mathrm{~h}$. Inhibition of cell proliferation by the two agents was measured by MTT assays. When administered simultaneously, clofarabine significantly enhanced gemcitabine sensitivity (as reflected in the decreased $\mathrm{IC}_{50}$ values) in all the cell lines (Fig. 1 and Table I). The combined effects of gemcitabine with clofarabine on cell proliferation were clearly synergistic, as determined by standard isobologram analyses (data not shown) and by calculating the $\mathrm{CI}$ values. A CI $<1$, indicative of synergism, was calculated for each of the drug combinations (Table I).

Effects of gemcitabine and clofarabine on cell cycle distribution in BxPC-3 cells. Since both gemcitabine and clofarabine are inhibitors of DNA synthesis, we hypothesized that gemcitabine combined with clofarabine would promote cell cycle arrest, resulting in synergistic cytotoxic effects. As shown in Fig. 2, treatment of BxPC-3 cells with $5 \mathrm{nM}$ gemcitabine alone resulted in $\mathrm{S}$ and $\mathrm{G} 2 / \mathrm{M}$ blockade, compared to untreated cells. Analogous results were obtained by treating the cells with $2.5 \mathrm{nM}$ clofarabine alone, however, to a much lesser extent. Notably, co-treatment with gemcitabine and clofarabine resulted in a nearly complete blockade from $\mathrm{S}$ to $\mathrm{G} 2$. These results demonstrated that clofarabine significantly augments $\mathrm{S}$ phase arrest induced by gemcitabine in BxPC-3 cells. Moreover, the presence of a hypodiploid pre-G1 region on the DNA histograms suggests that apoptosis was induced by gemcitabine alone and in combination with clofarabine (Fig. 2A).

Synergistic induction of apoptosis by gemcitabine and clofarabine in BxPC-3 cells. Efforts were then undertaken to further evaluate cell apoptosis induced by the two agents, as observed in Fig. 2A, by Annexin V/PI staining and flow cytometric analysis. Treatment with $5 \mathrm{nM}$ gemcitabine alone substantially induced apoptosis in BxPC-3 cells, whereas treatment with $2.5 \mathrm{nM}$ clofarabine at $2.5 \mathrm{nM}$ by itself only resulted in a marginal level of apoptosis (Fig. 3). When administered simultaneously, the two agents synergistically induced apoptosis, compared to individual drug treatments (cooperativity index $=0.67$ ).

Taken together, our results provide compelling evidence that the synergistic cytotoxic effects of the two agents against
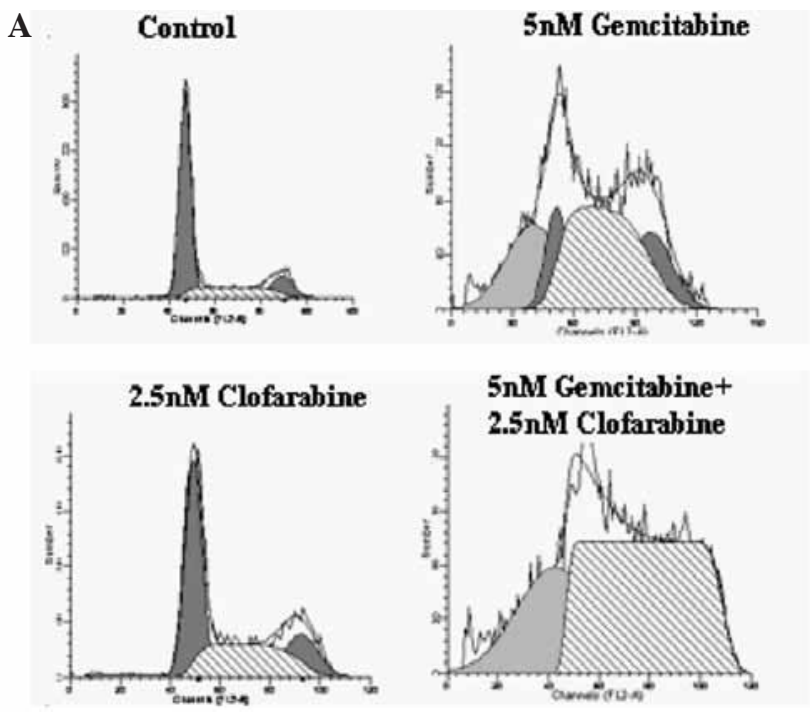

$\mathbf{B}$

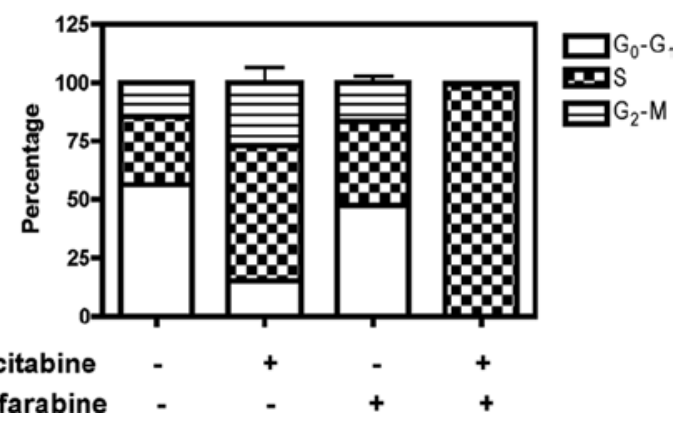

5 nM Gemcitabine -

$2.5 \mathrm{nM}$ Clofarabine $\quad-\quad+\quad+\quad+\quad+$

Figure 2. Effects of gemcitabine and clofarabine on cell cycle distribution in BxPC-3 cells. BxPC-3 cells were treated with $5 \mathrm{nM}$ gemcitabine or $2.5 \mathrm{nM}$ clofarabine alone or in combination for, $120 \mathrm{~h}$. Cell cycle distribution was determined by PI staining and flow cytometry. (A) DNA histograms from one representative experiment. (B) Average results from three to four independent experiments.

pancreatic cancer cells are attributed to induction of apoptosis and cell cycle arrest.

\section{Discussion}

Combining two nucleoside analogs is a potentially effective strategy to overcome drug resistance and to improve response and cure rates of cancer patients. Several reports describing positive interactions between purine and pyrimidine nucleoside 
A
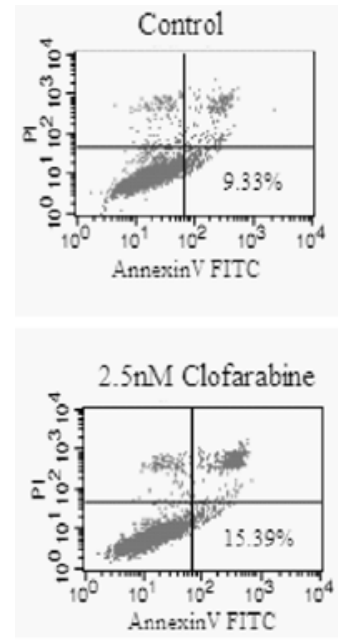

B

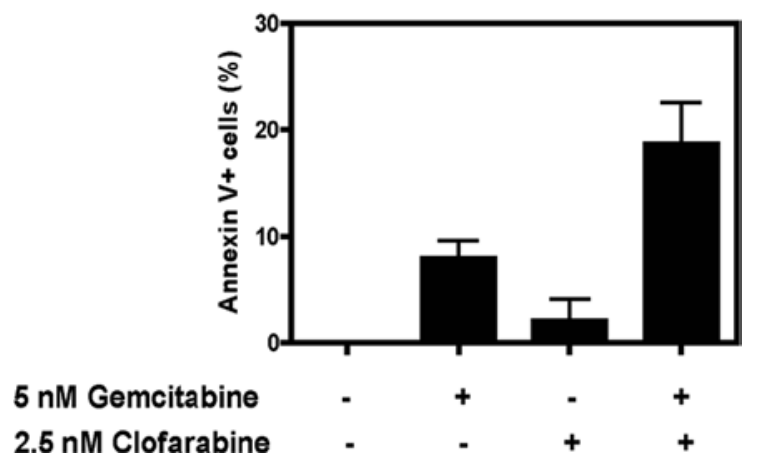

Figure 3. Clofarabine augments apoptosis induced by gemcitabine in BxPC-3 cells. BxPC-3 cells were treated with $5 \mathrm{nM}$ gemcitabine or $2.5 \mathrm{nM}$ clofarabine, alone or in combination for $120 \mathrm{~h}$. Early and late apoptotic events in the cells were determined by Annexin V/PI staining and flow cytometry. (A) Scatter plots of both early and late apoptotic events from one representative experiment. (B) Average results from three to four independent experiments.

analogs have focused on the treatments of leukemia or mesothelioma cells $(7,9,20,21)$, whereas treatments of pancreatic cancer cells with these combinations have not been reported. Our study demonstrated that the combination of gemcitabine, a pyrimidine nucleoside analog, and clofarabine, a purine nucleoside analog, resulted in synergistic cytotoxicities against three human pancreatic cancer cell lines. Most importantly, the concentrations of clofarabine required to enhance the cytotoxic effects of gemcitabine in the pancreatic cell lines were in the low $\mathrm{nM}$ range (1.25-10 $\mathrm{nM})$. This renders the feasibility of translating our findings into the clinic.

To begin the determination of the mechanisms underlying the synergistic cytotoxic effects of gemcitabine and clofarabine, cell cycle phase distributions were analyzed in post-drug treatment BxPC-3 cells. As expected, gemcitabine alone caused substantial accumulation of cells in the $S$ and G2/M phases, consistent with its mechanisms of action as a DNA chain terminator. Previous studies have also shown increased proportions of MIA PaCa-2, PANC-1 or A549 cells in $\mathrm{S}$ phase after treatment with gemcitabine $(22,23)$. Although clofarabine alone only marginally caused S and G2/M arrest, it substantially enhanced $\mathrm{S}$ phase arrest when simultaneously administered with gemcitabine. These results strongly suggest that $S$ phase arrest caused by the two agents clearly contributes to their synergistic cytotoxicities against pancreatic cancer cells. Similarly, apoptosis analysis showed that gemcitabine combined with clofarabine caused a synergistic induction of apoptosis in BxPC-3 cells, compared to individual drug treatments, suggesting that induction of apoptosis is indeed another contributing factor to the synergistic cytotoxicities of the two agents. However, detailed molecular mechanisms regarding how the agents synergize in causing $\mathrm{S}$ phase arrest and in inducing apoptosis remain unknown.

In conclusion, we showed that gemcitabine combined with clofarabine resulted in highly synergistic cytotoxicities against human pancreatic cancer cells mediated by $S$ phase arrest and apoptosis induction. The combination of gemcitabine and clofarabine could be effective against pancreatic carcinoma. Further studies to validate our in vitro results in vivo are warranted.

\section{Acknowledgements}

This study was supported by the Jilin University, Changchun, China, and the National Natural Science Foundation of China (no. 31071105).

\section{References}

1. Jemal A, Siegel R, Ward E, et al: Cancer statistics, 2008. CA Cancer J Clin 58: 71-96, 2008.

2. Burris HA III, Moore MJ, Andersen J, et al: Improvements in survival and clinical benefit with gemcitabine as first-line therapy for patients with advanced pancreas cancer: a randomized trial. J Clin Oncol 15: 2403-2413, 1997.

3. Kullmann F, Hollerbach S, Dollinger MM, et al: Cetuximab plus gemcitabine/oxaliplatin (GEMOXCET) in first-line metastatic pancreatic cancer: a multicentre phase II study. Br J Cancer 100: 1032-1036, 2009.

4. Li J and Saif MW: Advancements in the management of pancreatic cancer. JOP 10: 109-117, 2009.

5. Mini E, Nobili S, Caciagli B, Landini I and Mazzei T: Cellular pharmacology of gemcitabine. Ann Oncol 17 (Suppl 5): 7-12, 2006.

6. Giovannetti E, Danesi R, Mey V, Nannizzi S, Pasqualetti G and del Tacca M: In vitro studies on gemcitabine combinations with other antiblastics. Ann Oncol 17 (Suppl 5): 17-19, 2006.

7. Damaraju D, Damaraju VL, Brun M, et al: Cytotoxic activities of nucleoside and nucleobase analog drugs in malignant mesothelioma: characterization of a novel nucleobase transport activity. Biochem Pharmacol 75: 1901-1911, 2008.

8. Lech-Maranda E, Korycka A and Robak T: The interaction of gemcitabine and cytarabine on murine leukemias L1210 or P388 and on human normal and leukemic cell growth in vitro. Haematologica 85: 588-594, 2000.

9. Tosi P, Pellacani A, Zinzani PL, Magagnoli M, Visani G and Tura S: In vitro study of the combination gemcitabine + fludarabine on freshly isolated chronic lymphocytic leukemia cells. Haematologica 84: 794-798, 1999.

10. Zhenchuk A, Lotfi K, Juliusson G and Albertioni F: Mechanisms of anti-cancer action and pharmacology of clofarabine. Biochem Pharmacol 78: 1351-1359, 2009.

11. Bonate PL, Arthaud L, Cantrell WR Jr, Stephenson K, Secrist JA III and Weitman S: Discovery and development of clofarabine: a nucleoside analogue for treating cancer. Nat Rev Drug Discov 5: 855-863, 2006.

12. Faderl S, Gandhi V, Keating MJ, Jeha S, Plunkett W and Kantarjian HM: The role of clofarabine in hematologic and solid malignancies - development of a next-generation nucleoside analog. Cancer 103: 1985-1995, 2005.

13. Kantarjian HM, Jeha S, Gandhi V, Wess M and Faderl S: Clofarabine: past, present, and future. Leuk Lymphoma 48: 1922-1930, 2007.

14. Waud WR, Schmid SM, Montgomery JA and Secrist JA III: Preclinical antitumor activity of 2-chloro-9-(2-deoxy2-fluoro-beta-D-arabinofuranosyl)adenine (Cl-F-ara-A). Nucleosides Nucleotides Nucleic Acids 19: 447-460, 2000. 
15. Taub JW, Huang X, Matherly LH, et al: Expression of chromosome 21-localized genes in acute myeloid leukemia: differences between Down syndrome and non-Down syndrome blast cells and relationship to in vitro sensitivity to cytosine arabinoside and daunorubicin. Blood 94: 1393-1400, 1999.

16. Xie C, Edwards H, Xu X, et al: Mechanisms of synergistic antileukemic interactions between valproic acid and cytarabine in pediatric acute myeloid leukemia. Clin Cancer Res 16: 5499-5510, 2010.

17. Chou TC: Theoretical basis, experimental design, and computerized simulation of synergism and antagonism in drug combination studies. Pharmacol Rev 58: 621-681, 2006.

18. Tallarida RJ: Drug synergism: its detection and applications. J Pharmacol Exp Ther 298: 865-872, 2001.

19. Ten Cate B, Samplonius DF, Bijma T, de Leij LF, Helfrich W and Bremer E: The histone deacetylase inhibitor valproic acid potently augments gemtuzumab ozogamicin-induced apoptosis in acute myeloid leukemic cells. Leukemia 21: 248-252, 2007.
20. Chow KU, Ries J, Weidmann E, et al: Induction of apoptosis using 2',2' difluorodeoxycytidine (gemcitabine) in combination with antimetabolites or anthracyclines on malignant lymphatic and myeloid cells. Antagonism or synergism depends on incubation schedule and origin of neoplastic cells. Ann Hematol 79: 485-492, 2000.

21. Frewin RJ and Johnson SA: The role of purine analogue combinations in the management of acute leukemias. Hematol Oncol 19: 151-157, 2001.

22. Giovannetti E, Mey V, Danesi R, Mosca I and del Tacca M: Synergistic cytotoxicity and pharmacogenetics of gemcitabine and pemetrexed combination in pancreatic cancer cell lines. Clin Cancer Res 10: 2936-2943, 2004.

23. Giovannetti E, Mey V, Nannizzi S, et al: Cellular and pharmacogenetics foundation of synergistic interaction of pemetrexed and gemcitabine in human non-small-cell lung cancer cells. Mol Pharmacol 68: 110-118, 2005. 